\title{
A simulation and optimisation study: towards a decentralised microgrid, using real world fluctuation data.
}

Daniel Quiggin ${ }^{1}$, Sarah Cornell ${ }^{2}$, Mike Tierney ${ }^{3}$ and Richard Buswell ${ }^{1}$

1. Civil and Building Department, University of Loughborough

2. Stockholm Resilience Centre, Stockholm University

3. Queens School of Engineering, University of Bristol

Corresponding Author: Daniel Quiggin. D.Quiggin@lboro.ac.uk, Department of Civil and Building Engineering, Loughborough University, Loughborough, Leicestershire LE11 3TU, United Kingdom. Phone: +44 (0)7793107684, Fax: +44 (0)1509 223981

\begin{abstract}
A transition to a decentralised, decarbonised energy system for the domestic sector is constrained by the difficulty of obtaining energy balance between fluctuating demand and the intermittent, non-dispatchable power supply delivered by most renewables. A microgrid system including a mix of renewable generation technologies, energy storage and demand-response systems has been modelled using a linear programming approach, based on real-world data of residential energy consumption and weather variables. This model allows the exploration of the effects of fluctuations in demand and supply, microgrid scale and configuration, energy management options and alternative optimisation criteria. The model demonstrates quantitatively that a mixed-renewables microgrid system can reduce demand fluctuations and improve energy balance. Peak demand hour fluctuations were reduced by up to $19 \%$ for a simulated microgrid containing 144 households with one renewable unit and four batteries per household, with a renewables mix of $83 \%$ photovoltaic panels and $17 \%$ wind turbines. With this system, the demand on macrogrid energy supply was reduced by $16 \%, \mathrm{CO}_{2}$ emissions associated with energy use were reduced by $10 \%$ for all hours of operation, and by $74 \%$ during the hours of renewable supply. These findings suggest that microgrids using contemporary technologies can contribute significantly to $\mathrm{CO}_{2}$ mitigation targets.
\end{abstract}

\section{Keywords}

\section{Decentralised Energy Resources; Microgrid; Demand Response; Greenhouse Gases; Renewable Energy; Decentralised Energy}

\section{Introduction}

Anthropogenic emissions of greenhouse gases (GHG), in particular $\mathrm{CO}_{2}$, are accelerating climate change $[1,2]$. A major source of these $\mathrm{CO}_{2}$ emissions is the supply of energy from fossil fuels [1]. Climate mitigation, including meeting the commitments agreed under the UN Framework Convention on Climate Change, requires a radical decarbonisation of energy systems, with reduced dependence on fossil fuels, and accelerated diffusion of renewable technologies [3,4].

The current energy system is dominated by centralised generation, with electricity distributed to users through a macrogrid. The domestic sector accounts for a significant proportion of energy use; in 2006 the UK residential energy accounted for about $30 \%$ of final energy consumption and 35\% of all electricity consumed [5]. Decarbonising the residential electricity system presents particular challenges because the supply of energy from most renewable technologies is intermittent or stochastic in nature [6,7] due to 
their reliance on weather conditions (i.e. sunlight, wind). Consumer demand also fluctuates[8], exacerbating the problem. The electric load profile of the residential consumer is inherently more variable and 'peaky' than industrial or commercial sectors. The future national demand profile is projected to become increasingly peaky and stochastic, as large commercial and industrial loads turn to on-site generation [9]. This could leave the residential sector disadvantaged due to increased costs and reduced reliability.

To date, the focus of most research (and implementation) has been on domestic renewables embedded within the centralised-generation system. In other words, current efforts at transforming the energy system (shifting to generation by renewables) are still locked in to a dependence on a key component of the current system; the macrogrid. This study simulates an alternative energy system, consisting of an independent, decentralised microgrid made up of renewable, low GHG emission technologies. It starts from the premise that a demonstration of the effectiveness of such a system is a prerequisite to a reduced reliance on centralised generation systems.

\subsection{Distributed energy systems}

Energy generation can comprise many different types of technologies and involve renewable and nonrenewable energy sources [10-12]. The small-scale generation of electricity in close proximity to its end use is termed distributed generation (DG); the benefits and problems of which have been widely discussed [13]. The term embedded generation is used when DG is implemented in singularity within the centralised macrogrid system [14]. The macrogrid supplements energy supply during peak demand periods and the DG system supplies energy back to the macrogrid during high production periods. In contrast, a microgrid is a small-scale power (and often heat) network supplying many different loads with a variety of distributed energy resources in close proximity, controlled in order to balance supply and demand [15]. The benefits of aggregating distributed energy resources into a microgrid system include the increased scope for utilization of waste energy and efficiency gains as the DG units support local loads and are connected to many more loads $[12,16,17]$. A further benefit is that the microgrid has visibility within the macrogrid, allowing for reductions in the capacity of transmission-connected power stations, thus delivering climate mitigation gains [18].

Much of the current research $[12,13,16,19]$ has focused on microgrids operating in parallel to the macrogrid, being able to operate in 'islanded' mode for periods of time. The investigation by Abu-Sharkh et al. [15] is one of the few off-grid studies, and shows the feasibility of a combination of photovoltaics, micro-CHP and battery storage in enabling an independent microgrid.

\subsection{Energy balance issues in off-grid microgrids}

Meeting the energy demands of consumers is vital if a microgrid system is to be adopted, as consumers have grown accustomed to reliable and constant supply of electricity [11]. In the centralised macrogrid and grid-connected microgrid systems, fluctuations in demand result in frequency and reactive power issues [20], but supply can be increased or decreased to match demand because these systems access their energy from power-generating technologies that are controllable and dispatchable. Most renewable energy technologies are non-dispatchable and prone to intermittency, so fluctuation in supply and the matching of energy supply and demand present particular problems in DG systems $[21,22]$. An independent microgrid that meets all energy demands of consumers is necessarily more highly constrained than a microgrid connected to the macrogrid $[10,23]$. The independent microgrid will have a finite amount of available energy, fluctuating stochastically on many temporal scales depending on 
variables associated with the weather [15]. Thus in order to supply customers with energy from nondispatchable DG units, creative systems need to be developed [18].

Addressing the energy balance issues in a microgrid requires several approaches. Sovacool and Watts [24] propose eliminating energy balance issues by integrating renewable systems, coupling them to storage technologies and utilising 'smart meters' which allow two-way communication, enabling monitoring and control of the energy balance. Electrical power produced by one distributed energy resource that is not required locally can be fed into the microgrid and transferred to another that is unable to meet its demands. Currently, storage is only viable for small amounts of electricity over short time frames [11], although the technologies for doing so, such as lead-acid batteries, are well-established [25]. Demand Response (DR) mechanisms can also help to smooth the stochastic demand fluctuations $[15,26]$. Demand Response works by controlling the activity of appliances such as dishwashers, washing machines, fridges and freezers depending on the availability of electricity [8]. DR does not reduce total demand; rather, it flattens the fluctuations in demand, reducing the intensity of peak demand periods. Storage and DR can be combined with DG technologies to achieve greater energy balance [10,17]. Ren and Gao [27] investigated residential energy demands in Japan, looking at seasonal and short temporal fluctuations, and strongly emphasise the need for detailed knowledge of energy end-use or demand profiles in determining the number, capacity and operational strategy of distributed energy resources in order to achieve energy balance.

\subsection{Approach and rationale of this study}

At present, achieving overall energy balance in an independent microgrid is not considered to be feasible. Balance, however, can be improved by smoothing demand fluctuations and reducing the wastage from power supplied by intermittent renewables. This study has simulated a microgrid system that deploys multiple renewable technologies with DR and storage in order to minimise fluctuations in energy demand and optimise the energy balance. Within this study, peak demand is defined as hours with demand above average and it is these fluctuation periods that are minimised. This study does not represent an analysis of a complete energy system; in this case, other factors would need to feature in the analysis, including energy consumption reductions, which can be obtained through efficiency or energy conservation technologies [28].

The microgrid model allows the exploration of the effects of changing; grid scale (i.e. the numbers of households in the microgrid), configuration (units per household) and components (PV modules, wind turbines, storage batteries and DR), on the energy balance, net demand on the macrogrid and $\mathrm{CO}_{2}$ emissions reductions. The modelled microgrid has been set in a residential urban context. The high density of energy consumers in urban residential areas is an advantageous attribute for microgrids, enabling a high diversity of distributed energy resources to be implemented, which in turn facilitates energy balance despite the large variability in energy usage between households [29].

The carbon emissions associated with the deployment of the renewable energy units have been evaluated, but the complexity of this process needs to be recognised [30]. It should involve a full life cycle analysis (LCA; e.g., [20]), but there is still a shortage of data. The LCA-based emissions determined for the UK's current centralised macrogrid electricity system, consisting of a mix of fossil fuel, nuclear and renewable generation methods, have been estimated at $527 \mathrm{~g} \mathrm{CO}_{2}$-eq $\mathrm{kWh}^{-1}$ [31]. This value has been used as the baseline against which reductions are compared in this study. 


\section{Methodology and model implementation}

\subsection{Structure of the Model}

Figure 1 shows the structure of the model in terms of its main components, inputs and outputs.

MATLAB $^{\circledR}$ was used as the programming environment. In this study, two renewable technologies were deployed; solar photovoltaics (PV) and wind turbines. Many other technologies are available, but this simplified system gives an indication of the impact of implementing more than one technology and represents the most widely used technologies for domestic use at present. The modelled storage technology was lead-acid batteries.

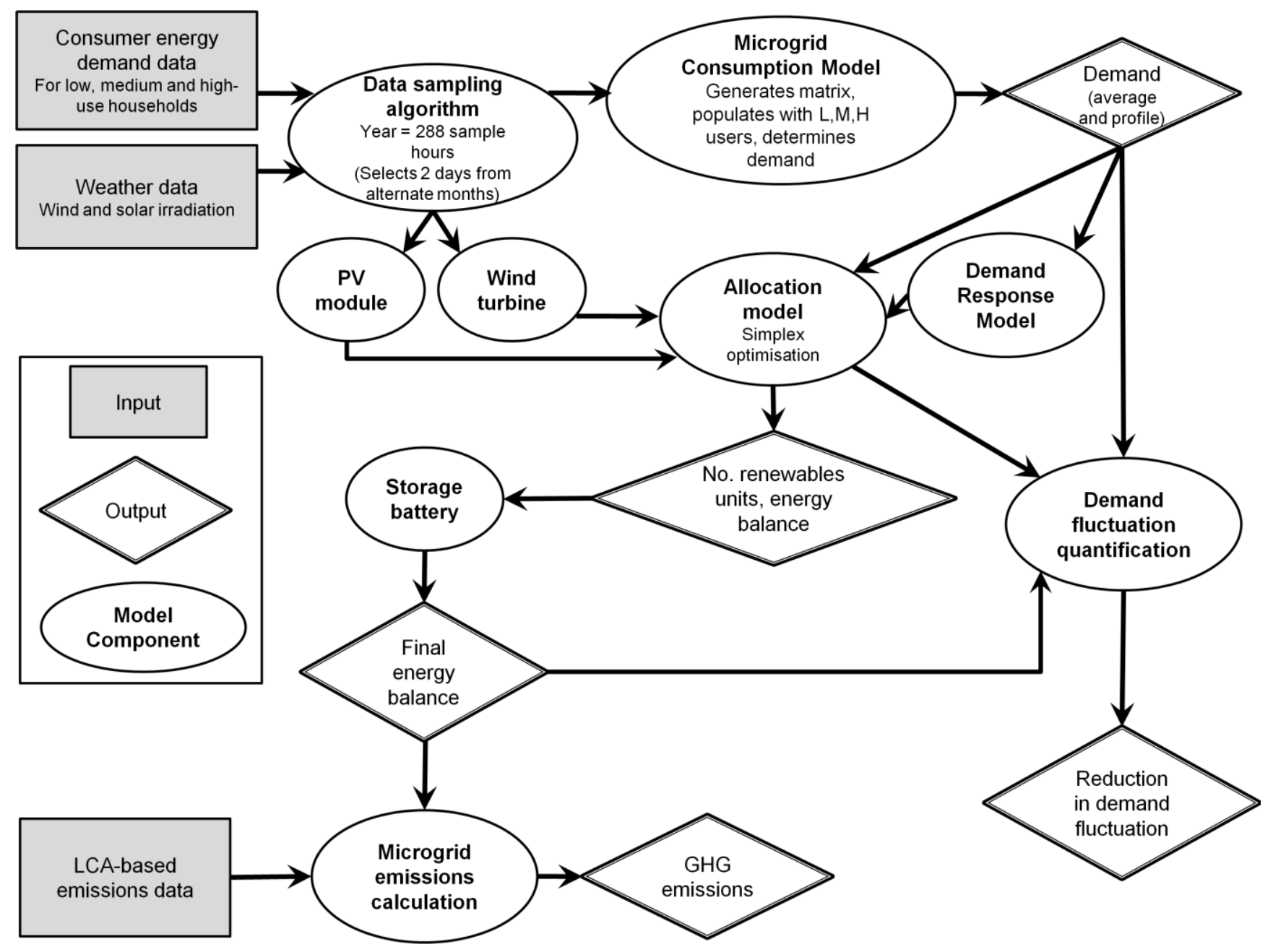

Figure 1: Flow diagram of the main components, input data and outputs of the microgrid consumption and renewable supply model.

The simulated system was designed to be realistically deployable in a normal UK city, so implementation data for real renewable units were obtained for use as inputs and constraints on the model. A typical residential wind turbine unit has an output of $1.5 \mathrm{~kW}$ [32]. In this study, data for the Swift Turbine unit [33] were used. A typical multi-panel solar photovoltaic unit has an output of roughly $1 \mathrm{~kW}$. To ensure similarity with the wind turbine unit, the model uses a solar PV system consisting of seven $200 \mathrm{~W}$ SX 3200B BP modules [34], with an area of $7 \times 1.43 \mathrm{~m}^{2}\left(\sim 10 \mathrm{~m}^{2}\right)$, an area used in other studies of the potential for PV deployment in the UK [35]. 


\subsubsection{Microgrid Consumption Model (MCM)}

The Microgrid Consumption Model (MCM) simulates a number of individual households populated by low, medium and high consumers (Table 1, Figure 2). The modelled microgrid size can be varied from a 4 $\mathrm{x} 4$ grid (16 homes) to $12 \times 12$ grid (144 homes) and so the relationships between grid size and fluctuations (expected to smooth due to averaging effects for larger grids) and between size and efficiency (as waste energy from one home can be used by another) can be investigated.

A random number generator populates each element of the grid with a low, medium or high consumer. The MCM combines the demand profiles of each individual home to determine the microgrid demand profile. The electricity usage data was taken from the COGEN database [36], obtained from three social housing sector homes in Newcastle (northeast England) and Llanelli (south Wales) and is representative of standard domestic European electricity use profiles. The original 5-minute data were summed into hourly time steps. The original data set encompasses a complete year (over 8760 hours), so a method proposed by Hawkes and Leach [10] has been applied to reduce the amount of data required in order to ease the computational load, while maintaining the impact of seasonal variation on the model. The method extracts one weekday and one weekend day from every second month (12 days in total), over a 12 month period resulting in 288 hours of demand profile data that ensures that seasonal variation is captured.

Because there are only three original demand profiles, if the same 12 days were selected for each low, medium and high consumer, an artificially peaky microgrid demand profile would be generated. In order to generate a more realistic microgrid demand profile, the MCM includes an algorithm that selects at random a different set of 12 days spread across the seasons for each individual household within the microgrid. Thus for each run of the model, a unique microgrid demand profile is generated.

\begin{tabular}{|c|c|c|c|c|c|}
\hline $\begin{array}{c}\text { Consumer } \\
\text { Type }\end{array}$ & $\begin{array}{c}\text { Annual } \\
\text { Consumption } \\
{[\mathrm{kWh}]}\end{array}$ & Location & Year & $\begin{array}{c}\text { Size of } \\
\mathrm{dwelling} \\
{\left[\mathrm{m}^{2}\right]}\end{array}$ & Occupancy type \\
\hline Low & 1155 & Newcastle & 2005 & 65 & Single male \\
\hline Medium & 3028 & Newcastle & 2005 & 65 & Mother and two children \\
\hline High & 8387 & Llanelli & 2003 & 108 & Mother and five children \\
\hline
\end{tabular}

Table 1: Household characteristics of the low, medium and high consumers modelled 


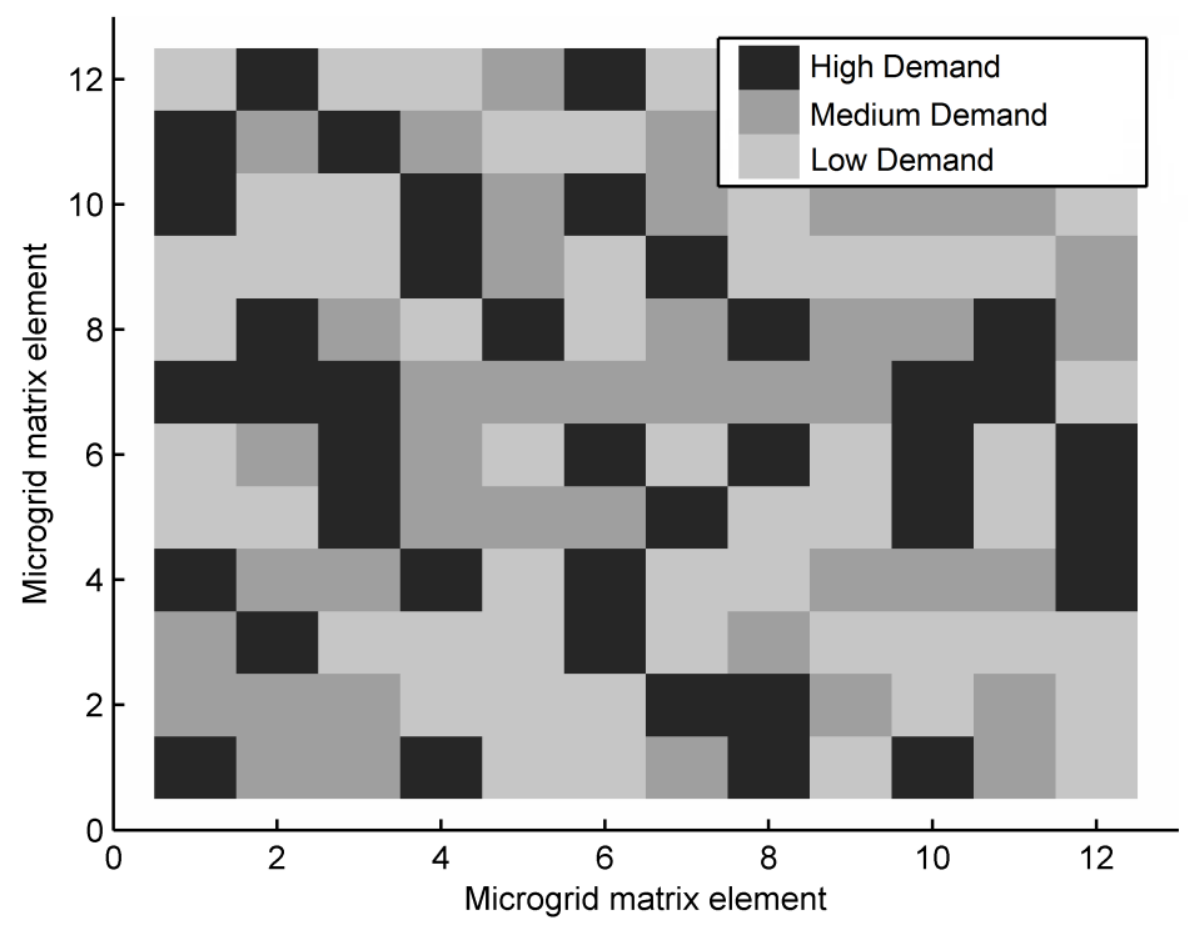

Figure 2: a) Example 12 x 12 microgrid configuration of low, medium and high consumers corresponding to light grey, grey and black matrix elements respectively.

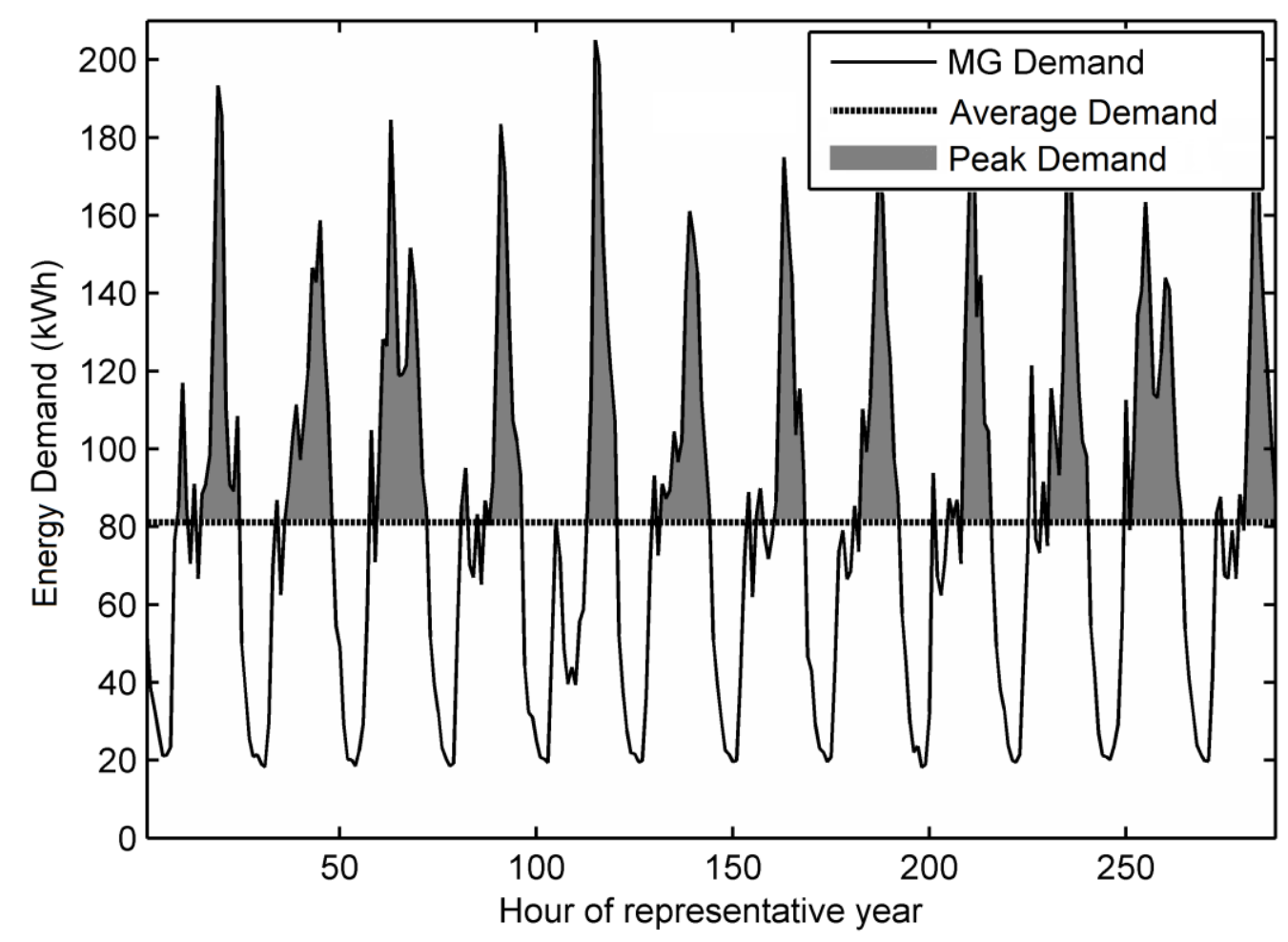

Figure 2: b) Microgrid consumption model demand profile output for a 12 x 12 microgrid over the 12 sampled days (288 hours). 


\subsubsection{Solar PV Model}

The solar PV model used in this study was that of Walker [37], where a complete description can be found. Here the model assumes a direct connection between the PV module and batteries with a series blocking diode, whereas Walker used a Maximum Power Point Tracking (MPPT) inverter. This results in a conservative estimation of the PV output yields, lower than would be achieved with a MPPT inverter. Specification details were obtained from the technical literature of the BP SX 3200B 200W PV Module [34]. The model is based on the Shockley diode equation. The model has a temperature-dependent photo-current source, $I_{L}$, a temperature-dependent series resistance, $R_{S}$, and a diode junction with a temperature-dependent saturation current of the diode, $I_{0}$. A single shunt diode was modelled, the diode quality factor set to achieve the best IV curve match.

The power output of the PV unit is dependent not only on the solar irradiation but also the temperature of the module and the voltage across its terminals. The IV curve was conservatively assumed to be flat until 13V, after which temperature starts to impact significantly on the IV relationship. A $12 \mathrm{~V}$ battery was used as the output terminal voltage. As the demand from the microgrid is $240 \mathrm{~V} \mathrm{AC}$, a $70 \%$ efficiency was assumed for the required inverter, which is also a conservative approach yielding a lower output than could be achieved with a high performace inverter.

Temperature and solar irradiation data were obtained from the Met Office MIDAS Land Surface Station at Milford Haven [38]. This is an urban site, imperative for any study of urban microgrids. It is also near to Llanelli, the location of one of the demand profiles. Weather data from 2006 which correlated to the year the energy consumption data was gathered. Direct radiation data from Milford Haven was unavailable which meant the separation of diffuse and direct radiation within the global radiation data was not possible. The PV units were therefore modelled on the horizontal plane. As for the MCM, the Solar PV model includes an algorithm that pulls 12 days of weather data at random from alternate months from a full year's data. The 12 days are not necessarily the same as those selected by the MCM. In this way, the weather data and consumption data correspond to the same seasonal variation.

\subsubsection{Wind Turbine Model}

Using the approach described by Mithraratne [39], the output profile of the Swift turbine [33] was utilised alongside a turbine efficiency of $37 \%$ to convert wind speeds into $240 \mathrm{~V}$ AC power output. The model accounts for the reduction in wind speed and turbulence due to changes in surface roughness in the built-up urban environment (Equation 1; [40,41]).

$$
v_{u}=v_{o} \cdot\left(\frac{z_{u}}{l_{u}}\right)^{\alpha_{u}} /\left(\frac{z_{o}}{l_{o}}\right)^{\alpha_{o}}
$$

$v_{u}$ is the urban wind velocity at a height $Z_{u} ; v_{o}$ is the open ground or weather-station wind velocity taken at a height $Z_{o} ; \alpha$ is an empirical exponent, which depends on the surface roughness, stability and temperature gradient. The urban exponent, $\alpha_{u}$, was set to 0.22 , which is consistent with urban areas with closely spaced obstructions with at least $2000 \mathrm{~m}$ of similar terrain in all directions. The open ground exponent, $\alpha_{o}$, was set to 0.14 , consistent with open terrain scattered with obstructions less than $10 \mathrm{~m}$ in height, representative of the weather station environment. $l_{u}$ and $l_{o}$ are the urban and open ground layer thickness, respectively, which is the height at which the gradient velocity is first observed.

Wind speeds at a height of $10 \mathrm{~m}$ were also obtained from the Milford Haven MIDAS Station [38]. A height of $10 \mathrm{~m}$ is typical for a wind turbine in an urban residential setting [42]. As for the PV and MCM models, the wind turbine model selects 12 random days across the seasons from the year's data. 


\subsubsection{Storage Model}

Lead-acid batteries are currently the most suitable technology for short-term electrical storage. The approach of Jenkins et al. [43] has been applied to model the state of charge (SOC), power charging and discharging behaviours. For each run of the model, the batteries start with zero SOC, to prevent external power entering the microgrid. Because our focus was on minimising fluctuations above average demand, the batteries were preferentially set to charge and discharge in relation to the average demand.

Power surplus, $P^{+}$, occurs when power generated exceeds demand, or when power is generated while demand is below average. Under these conditions, the system charges the batteries, subject to the constraint that the SOC of the batteries must not exceed rated capacity. The charge current, $I_{c}$, is given by Equation 2:

$$
I_{c}=P^{+} \cdot \varepsilon_{c c} \cdot \varepsilon_{c} / V_{b}
$$

$V_{b}$ is the battery voltage, $12 \mathrm{~V}$; the charge controller efficiency, $\varepsilon_{c c}=98 \%$; and the AC/DC convertor efficiency, $\varepsilon_{c}=80 \%$. The charge current must be less than the maximum charge current allowed by the batteries.

Power shortfall, $P^{-}$, occurs when demand is greater than both average demand and generation. Discharge current is dictated by the power shortfall and the SOC of the battery. The SOC reduces, but must not fall below $20 \%$ rated capacity. The required discharge current is given by Equation $3 . \varepsilon_{i}$ is the efficiency of the DC/AC inverter.

$$
I_{D}=P^{-} / V_{b} \cdot \varepsilon_{c c} \cdot \varepsilon_{i}
$$

The discharge efficiency, $\beta$, is defined by the following empirical relationship [43]:

$$
\beta=\left(13.3 \cdot \ln \left(C_{b} / I_{D}\right)+59.8\right) / 100
$$

where $C_{b}$ is the maximum capacity of the battery system. The change in charge at any point in time is then given by Equation 5:

$$
\Delta C=I_{c} \cdot \Delta t-\left(I_{D} \cdot \Delta t / \beta\right)
$$

\subsubsection{Demand Response Model (DRM)}

Moura and de Almeida [26] have quantified the effect of demand response on energy demand, and suggest that within each 24 hours the peak demand hour can be reduced by $8.3 \%$. This empirical assessment has been implemented in the DR model by shifting this proportion of peak loads to an hour where demand falls below the value of Euation 6, and that lies within \pm 2 hours of the peak demand hour. This latter constraint prevents unrealistic deferrals of energy use.

$$
D \leq \frac{\left(D_{p e a k}-D_{a v}\right)}{2}+D_{a v}
$$




\subsection{Optimisation of renewables mix in the microgrid}

Several studies $[10,27]$ have investigated the optimisation of the high level design of microgrids with distributed generation and storage. Planning and optimising the dispatch or operational schedule of DG in meeting demand, the unit commitment problem, is generally based on economic costs. Here, the target optimisation is the minimisation of demand fluctuations by utilising the mix of renewable technologies. This gives an indication of which renewables are best suited to a particular demand profile [10].

Linear programming has been used for unit commitment applications [10,44]. It represents prescribed system requirements as linear equations, which impose constraints within the simplex algorithm $[45,46]$. Here, both linear equalities and inequalities define a polytopic feasible region, the edges of which are moved by the simplex algorithm towards the optimum solution. The formulation of the linear problem is given below.

$$
\begin{aligned}
& \max _{x} f(x) \quad \text { subject to } \quad A x \leq b \\
& A_{e q} \cdot x=b_{e q} \\
& l b \leq x \leq u b
\end{aligned}
$$

The function $f(x)$ is the output of the renewable technologies during each (sampled) hour of the typical year, where $x$ represents the number of renewable units. The optimisation determines the number of units of each technology type that results in the greatest demand fluctuation reduction. Each household in the microgrid is constrained to have an integer number of technologies (i.e., for a $4 \mathrm{x} 4$ microgrid in which each household has one renewables unit, the optimisation must select an integer number of wind turbines and solar PV modules totalling a maximum of 16 units). $b_{e q}$ is the total number of units in the microgrid and $A_{e q}$ constrains the configuration of units to be constant throughout the year. Information regarding the demand to be met is contained within the inequality constraint vector $b$, in which each element represents one of the 288 hours (see section 2.1.1), and the hourly output of each technology is contained within the inequality matrix $A$. The upper bound, $u b$, of $x$ is set to the total number of units in the microgrid and the lower bound, $l b$, is set to zero. The output of this optimisation is to select an integer number of solar PV panels and wind turbines which give the greatest demand fluctuation reduction. Once the optimal number of modules is set, demand is reduced by the output profile of the configuration chosen by the optimisation.

The generalised objective function becomes;

$$
f(x)=\sum_{i=1}^{288}\left(S_{i} \cdot x^{s}+W_{i} \cdot x^{w}\right)
$$

A. $x \leq b$ becomes :

$$
S_{i} \cdot x^{s}+W_{i} \cdot x^{w} \leq D_{i}
$$

$A_{\text {eq }} \cdot X=b_{\text {eq }}$ becomes:

$$
x^{s}+x^{w}=N
$$

$l b \leq x \leq u b$ becomes: 


$$
0 \leq\left(x^{s}, x^{w}\right) \leq N
$$

Where $i$ is the hour of the typical year, $S_{i}$ and $W_{i}$ are the solar PV and wind turbine units output each hour of the typical year, $x^{s}$ and $x^{w}$ are the optimal number of solar PV and wind turbine units as defined by the optimisation. Further to this, $D_{i}$ is the demand each hour of the typical year and $N$ is the total number of renewable units per household multiplied by the number of households. The convergence criteria was set at $10^{-7}$ for the simplex algorithm, the runtime ranged between 1.7-2.0 seconds per optimisation.

This optimisation can be performed for all hours of the year, or for peak hours by disregarding hours when demand is below average. The main focus of this study is on peak demand hour optimization because peak-hour fluctuations are more difficult to supply and in the simulated system, the DRM and storage models both address only the peak hours. However, it is also important to assess the system's overall change in requirements for energy from the macrogrid and its $\mathrm{CO}_{2}$ mitigation potential, so allhours optimisations are also reported in some of the discussion that follows.

\subsection{Quantifying Demand Fluctuations}

There is no standard metric for describing demand or supply fluctuations in relation to an independent power system. For a centralized macrogrid, energy balance is not problematic as averaging over large numbers of users smoothes fluctuations. The literature on reliability and stability of power systems is therefore primarily concerned with frequency and reactive power deviations. Kirby and Hurst [47] used the coefficient of variation when comparing industrial to non-industrial load profiles. We have opted to use standard deviation as the demand fluctuation metric, which is both simple to determine and conceptualise. Although the weather and energy consumption data show log-normal distributions, the sampling techniques and data handling in the demand response, renewables unit and storage models result in more symmetric distributions for the output data, so a normal distribution is assumed to be an adequate approximation for the purposes of this study. There is no need to reduce demand fluctuation when usage is low (i.e. below average demand) and supply unconstrained, so in this study, the quantitative measure of interest is the standard deviation of demand fluctuations above the average demand.

In order to assess the most important factors affecting demand fluctuation reduction, a set of scenarios were defined that varied the demand response model, the diversity of renewable technologies, storage and scale of microgrid.

\subsection{Estimating the microgrid's GHG emissions}

In order to assess the GHG mitigation potential of the microgrid system, emissions associated with each unit in the grid have been estimated, on an LCA basis where possible:

- The $1.5 \mathrm{~kW}$ Swift wind turbine has emissions of $121 \mathrm{~g} \mathrm{CO}_{2}$-eq $\mathrm{kWh}^{-1}$ [48], assuming the turbine is not recycled;.

- No LCA value was available for the BP SX 3200B PV unit, so a value of $72.4 \mathrm{~g} \mathrm{CO}_{2}$-eq $\mathrm{kWh}^{-1}$ for a similar unit was used [49].

- The lead-acid batteries have the greatest associated emissions, at $180 \mathrm{~g} \mathrm{CO}_{2}$-eq $\mathrm{kWh}^{-1}$, derived from a number of sources [50-52].

Multiplying these emissions by the number of kilowatt-hours of operation for each unit and summing over the hours of operation gives the microgrid's total emissions per year. 


\section{Results and analysis}

\subsection{Subcomponent model outputs}

With the exception of Figure 8, figures in this section show sample results for $12 \times 12$ microgrids, although grids ranging in size from $4 \times 4$ to $12 \times 12$ were modelled. Figure 2a shows an example microgrid household configuration created by the MCM, and Figure $2 b$ shows an example microgrid energy consumption profile generated by the MCM by sampling 12 days of consumption data for each category of household. By determining the probability distribution (log-normal) of the energy consumed, average demand was calculated and peak hours identified (shown in Figure $2 \mathrm{~b}$ as shaded areas above the average demand line).

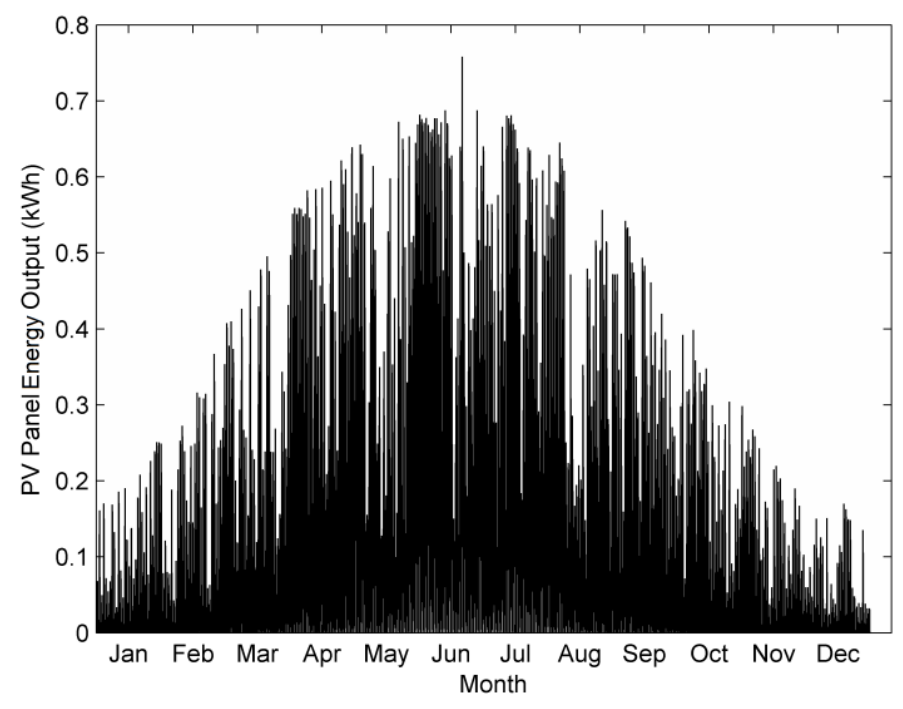

Figure 3: a) Energy generated throughout the year for the modelled PV system based on solar irradiation data from Llanelli

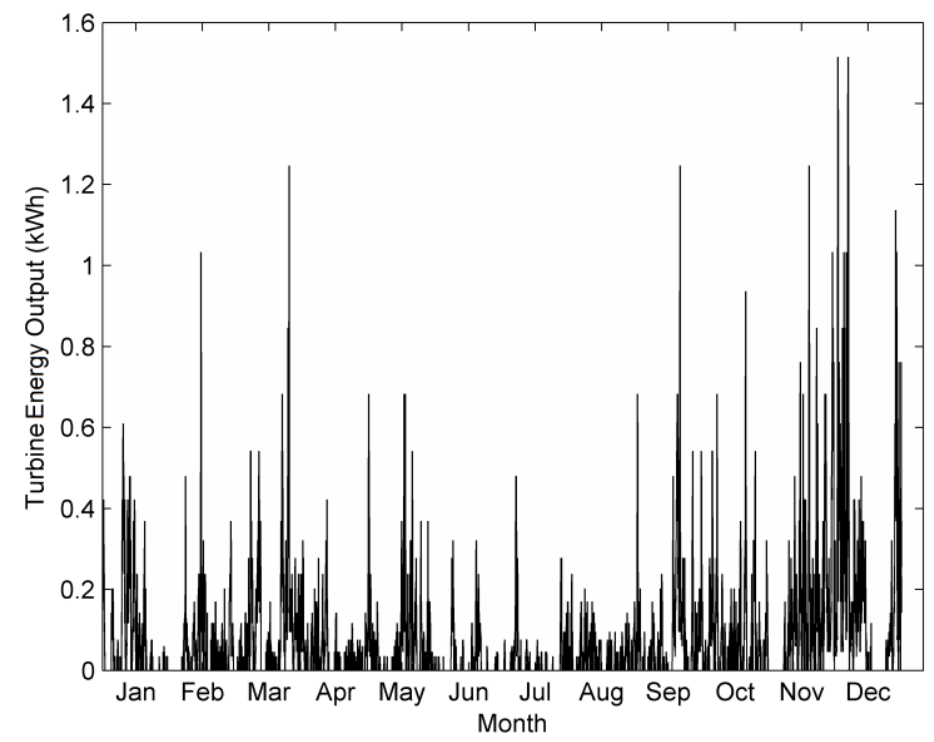

Figure 3: b) Energy generated throughout the year for the modelled wind turbine system based on wind speed data from Llanelli. 
Figures $3 \mathrm{a}$ and $3 \mathrm{~b}$ show the power generated through the model year by the PV module and wind turbine unit. In the simulated system, the PV module generates an average output of $2.21 \mathrm{kWh}$ per day (somewhat lower than a previous estimate [35] of $5 \mathrm{kWh}$ per day for $10 \mathrm{~m}^{2}$ of roof mounted PV panels). The output from the wind turbine model averages $1.72 \mathrm{kWh}$ per day, consistent with the range of $1.44-$ $2.30 \mathrm{kWh}$ per day reported by Mithraratne [39], but much higher than Mackay's [35] value for roofmounted urban wind turbines of $0.2 \mathrm{kWh}$ per day. This divergence in the literature may be attributed to the extremely intermittent nature of wind turbine output, which has a highly skewed distribution, with many days with minimal output. Figure 4 shows PV module and wind turbine outputs for an indicative 12 sample days of operation. The PV module output is more reliable but smaller in magnitude than the turbine output.

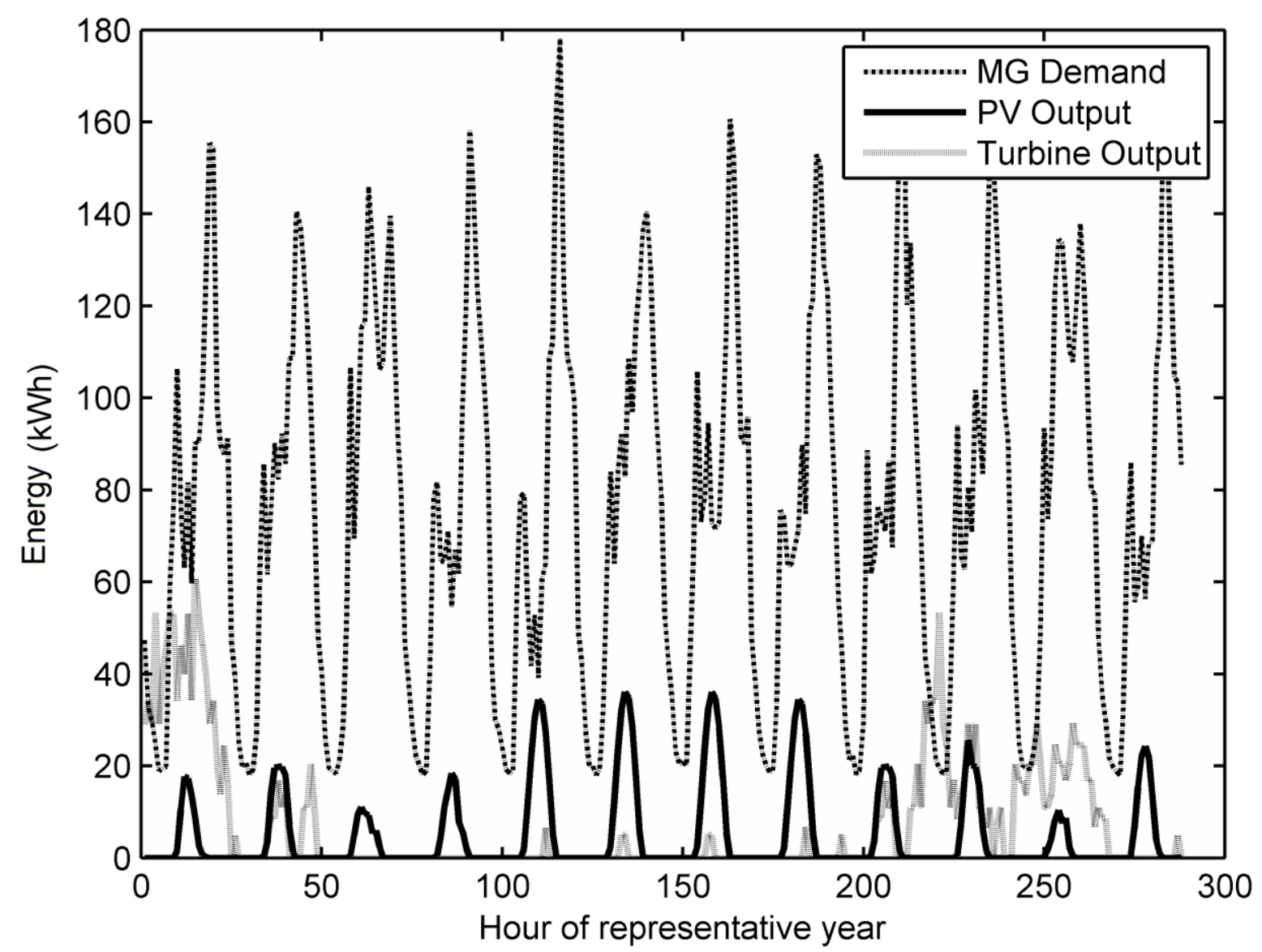

Figure 4: Output from the PV panel and wind turbine systems in a $12 \times 12$ microgrid where the total system comprises 144 turbines and 144 PV panel systems. 


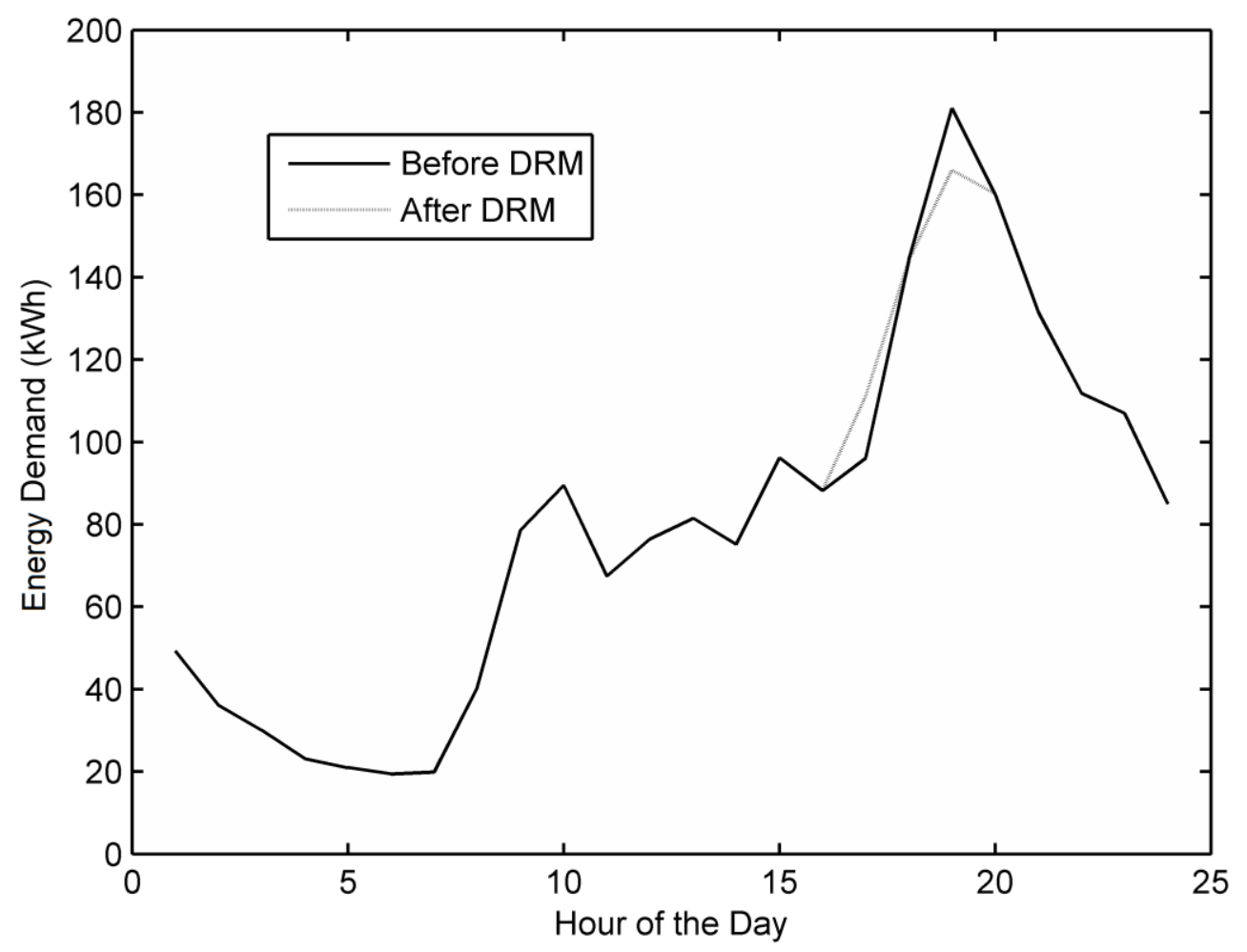

Figure 5: Average 24 hour demand of the $12 \times 12$ microgrid before and after demand reduction model.

Figure 5 shows the average simulated 24-hour demand profile of the microgrid. The shape of the profile is very similar for all sizes of microgrid, and also similar to the average residential UK electricity demand profile. Figure 5 also illustrates how within each 24 hour cycle, the DRM reduces demand in the peak demand hour by $8.3 \%$, by shifting demand temporally by up to 2 hours (i.e., the total system energy remains constant).

The battery storage model also produced realistic results (Figure 6). Energy generated when demand is below average charges the batteries, which are then discharged when demand rises beyond average demand. If renewable energy generation does not exceed demand, then due to inefficiencies in DC/AC power conversion, the total system should embody less energy with increasing battery use. This was observed in the simulation results. 


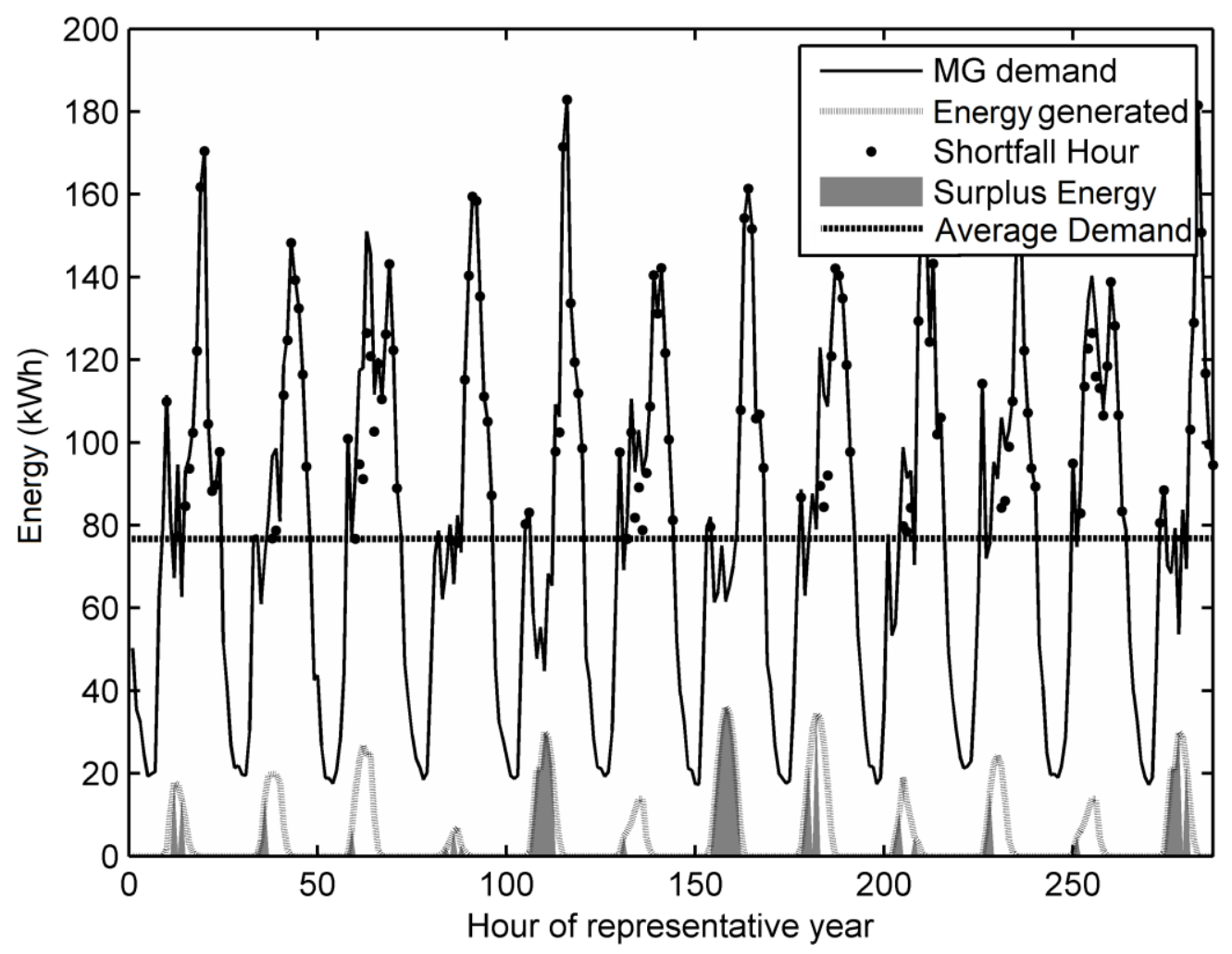

Figure 6: An example of the allocation of surplus and shortfall hours for charging and discharging the batteries, performed with a $12 \times 12$ microgrid

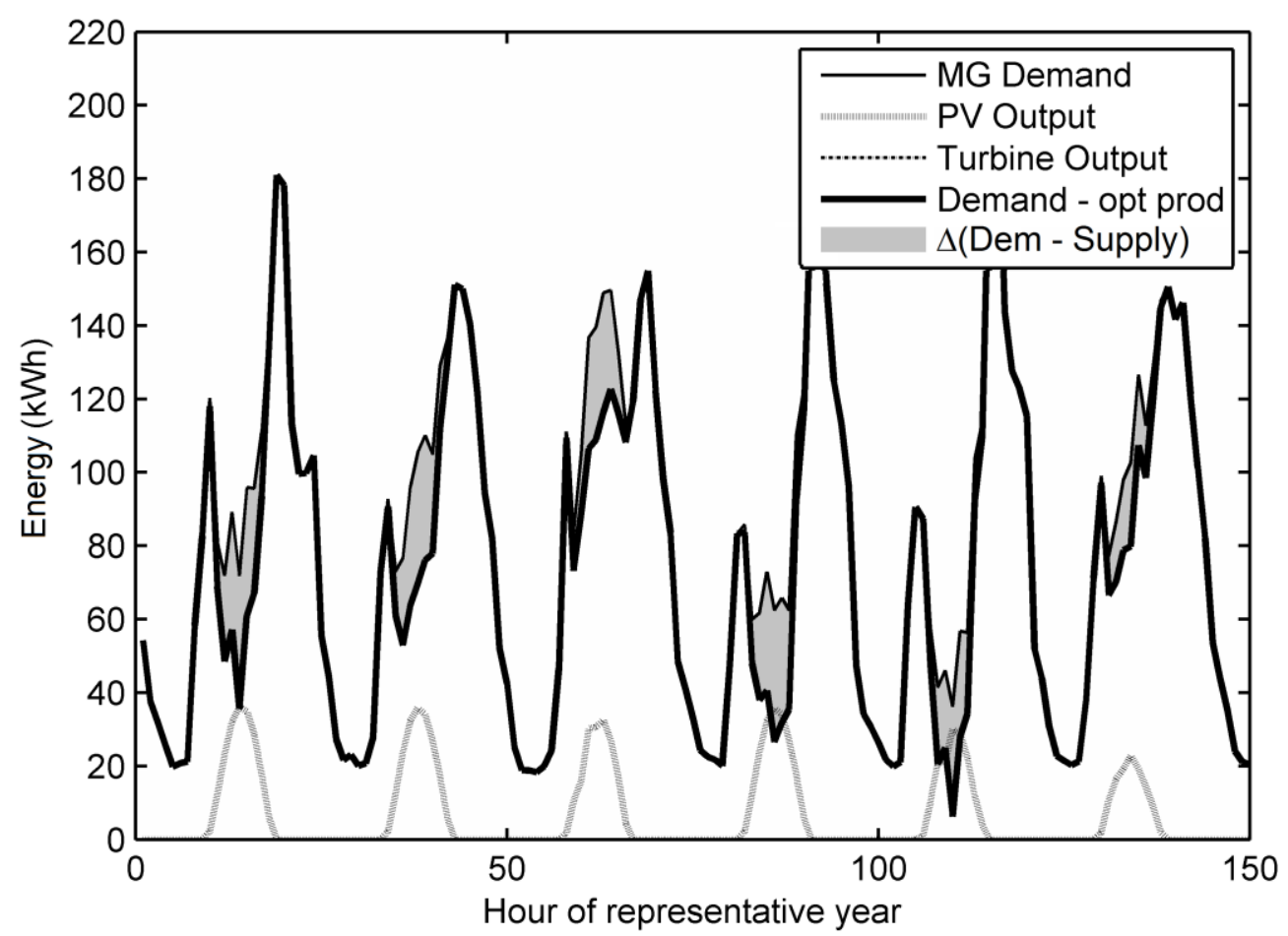

Figure 7: a) Microgrid demand and renewable output at optimal levels, optimisation performed for peak demand hours (12 x 12 grid) 


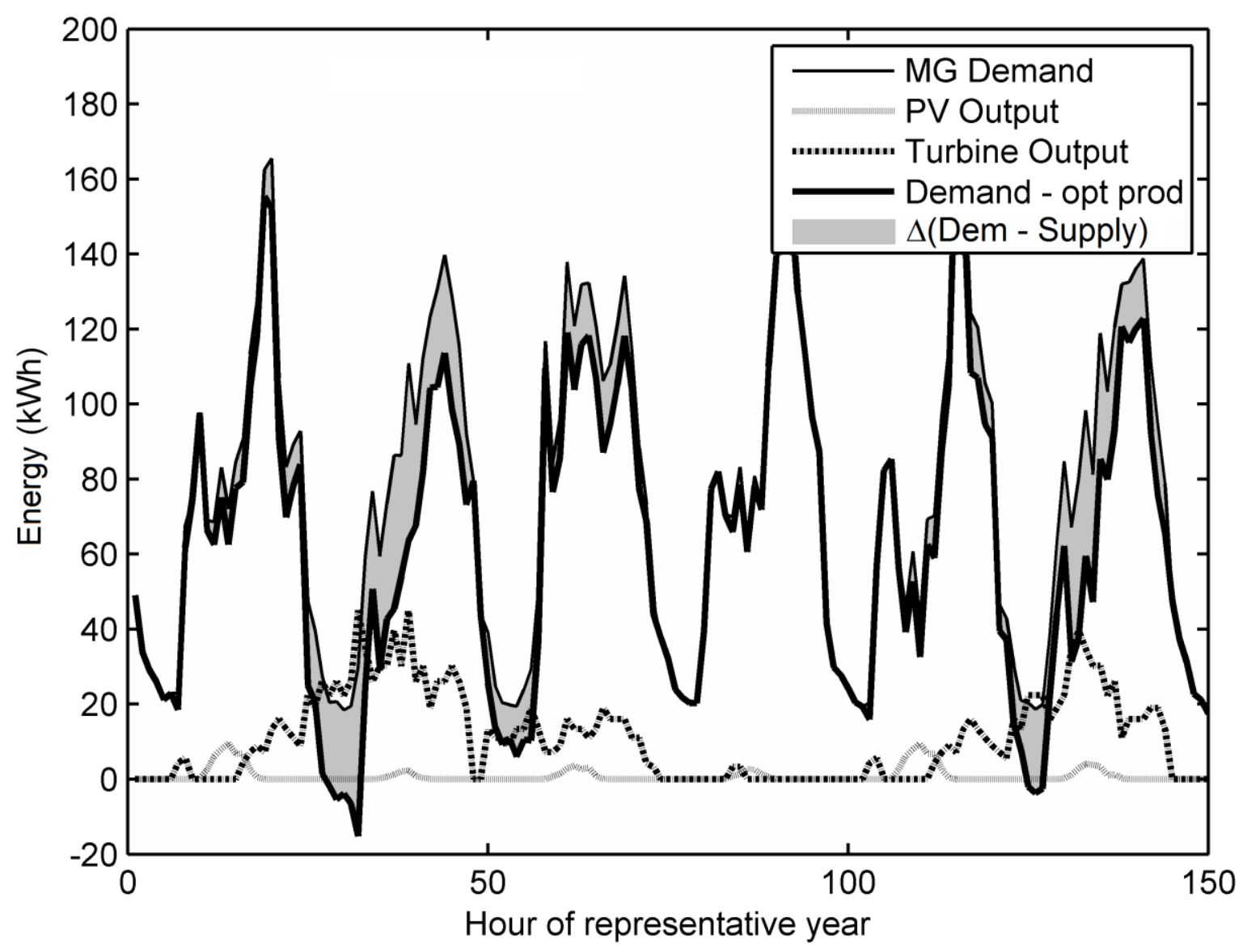

Figure 7: b) Microgrid demand and renewable output at optimal levels, optimisation performed for all hours (12 x 12 grid)

Figure 7 shows example runs that demonstrate the functioning and dynamic behaviour of different components of the system for a 12 x 12 microgrid (just the first 150 of the 288 sampled hours in the year are shown, for the sake of image clarity). With one renewable unit per household and no battery storage, the peak hours optimisation (Figure 7a) allocated all generation to PV modules. This outcome is likely due to the strong correlation peak demand times with PV module output, which is highest in the middle of the day when sunlight is brightest. For the optimisation performed for all demand hours (Figure 7b), the optimisation returns a mixture of PV modules and wind turbines.

Figure 8 illustrates the effect of including storage batteries in the system. Without batteries, when the renewable units generate a large amount of power while demand is low, the energy is wasted (Figure 8a). Figure $8 \mathrm{~b}$ shows how batteries contribute to the fluctuation reduction, by discharging stored energy back into the system during peak demand. 


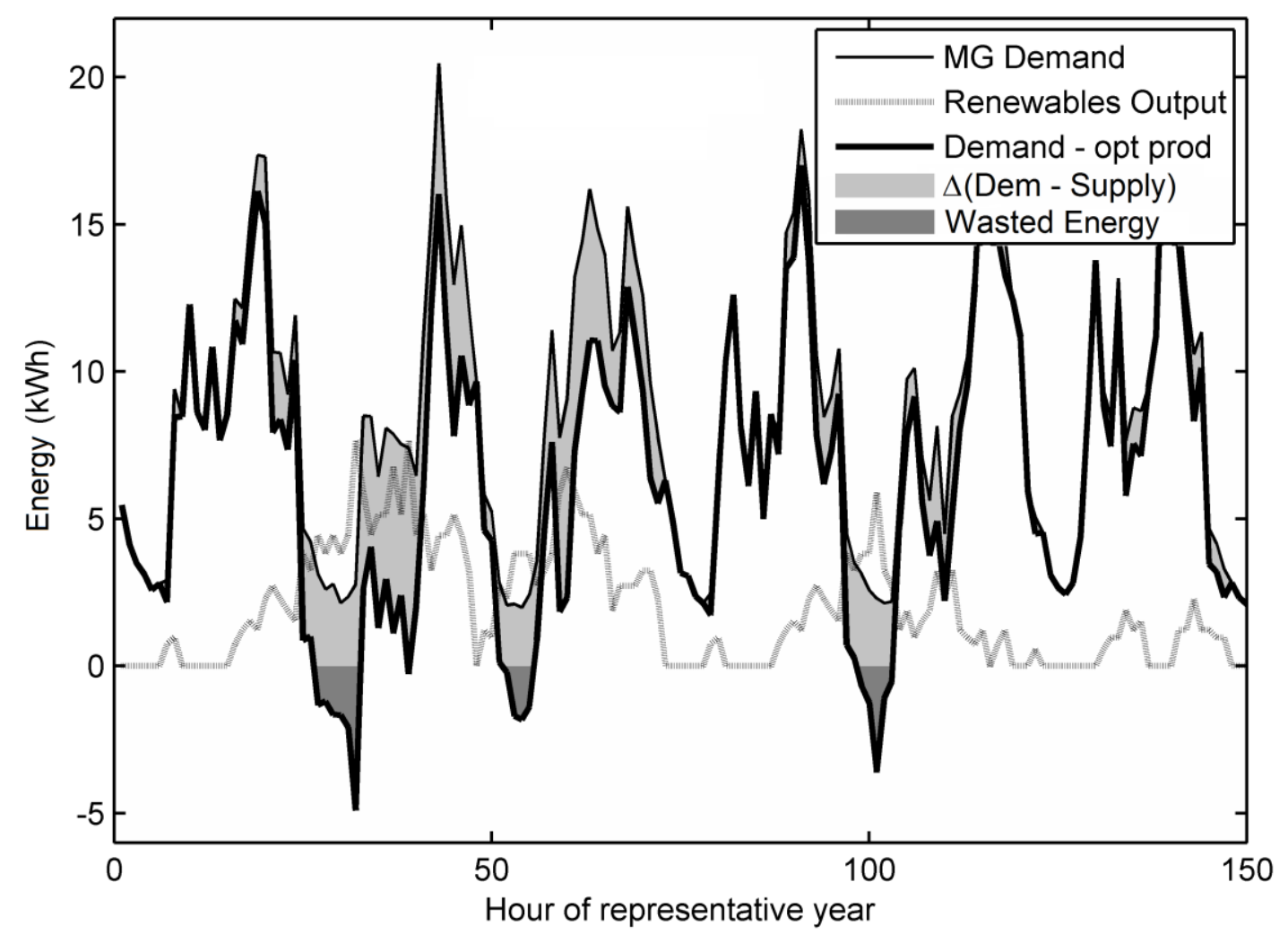

Figure 8: a) Microgrid demand and renewable output at optimal levels, optimisation performed for all demand hours with no batteries to store energy when supply > demand ( 4 x 4 grid)

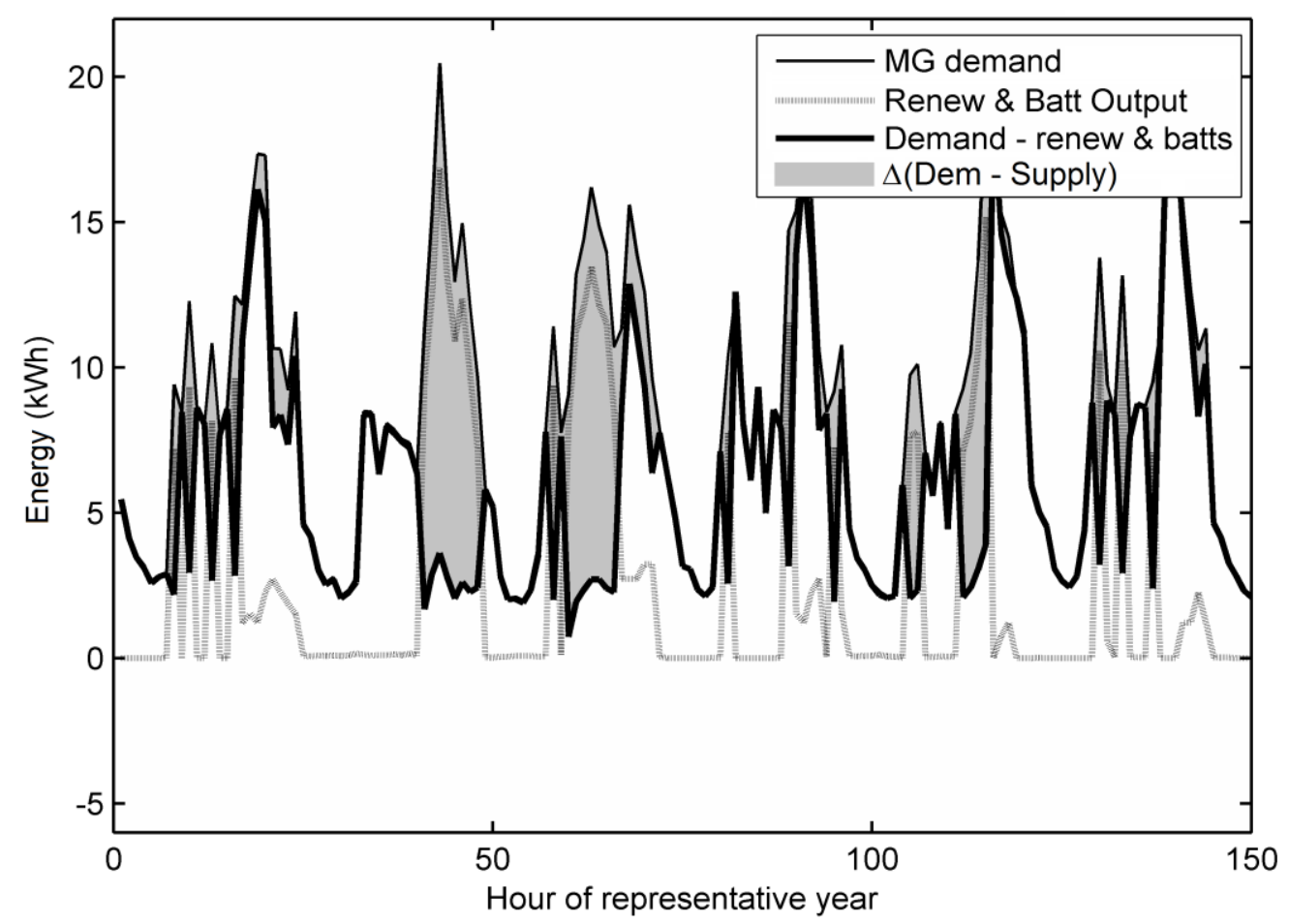

Figure 8: b) Microgrid demand and renewable output at optimal levels, optimisation performed for all demand hours with batteries to shift generated energy to peak demand hours. ( $4 \mathrm{x} 4 \mathrm{grid}$ ) 


\subsection{Sensitivity analyses}

\subsubsection{Variations in the data}

The most variable and influential factors in the model are the weather and consumption data, as they are stochastic input variables in a deterministic model. The sensitivity of the output associated with the variability in input data has been quantified using a Monte Carlo (MC) sampling technique. The model was run 1000 times, with a different set of inputs randomly sampled for each run from the input parameter distribution space (i.e., the lognormal distributions of weather and consumption data). This produces a probability distribution for each output of the model. Accordingly, in the discussion and figures of the model output, one standard deviation $(1 \sigma)$ on the mean of the distribution is given.

\subsubsection{Effect of microgrid size}

The size of the microgrid does not have a significant impact on the system's ability to reduce demand fluctuations. It was initially expected that efficiency gains would be evident as wasted energy due to occupancy variation would decrease. In fact, there is no evidence in this simulated system of a smoothing, averaging effect of increasing sample size. On a per-household basis, the demand fluctuation before implementation of renewable units, batteries or DRM is the same, at $(0.2 \pm 0.02) \%$ per household, for the $4 \times 4$ grid (16 households) and the $12 \times 12$ grid (144 households). This is likely a function of the demand profiles and highlights the limitations of this simulation model in light of real household data shortage. The total demand fluctuation reduction by the whole system remains close to constant, at around $16 \%$, as microgrid size increases from $4 \times 4$ to $12 \times 12$. However, as microgrid size grows, the renewable units contribute proportionally more to the reduction (Figure 9), rising from (10.8 \pm 1.2$) \%$ for a $4 \times 4$ grid to about $(12.0 \pm 1.4) \%$ for grids greater than $8 \times 8$. The contribution to demand fluctuation reduction made by storage batteries decreases from $(3.9 \pm 0.4) \%$ to $(3.5 \pm 0.4) \%$, and the demand response shifts also show a small decrease with increasing grid size.

Microgrid size also shows no consistent effect on the model outputs: the optimal system configuration, $\mathrm{CO}_{2}$ emission mitigation, and macrogrid energy supply reduction remain approximately constant (Figure 9). Accordingly, for simplicity, in this discussion, results shown relate to a 12 x 12 microgrid. 


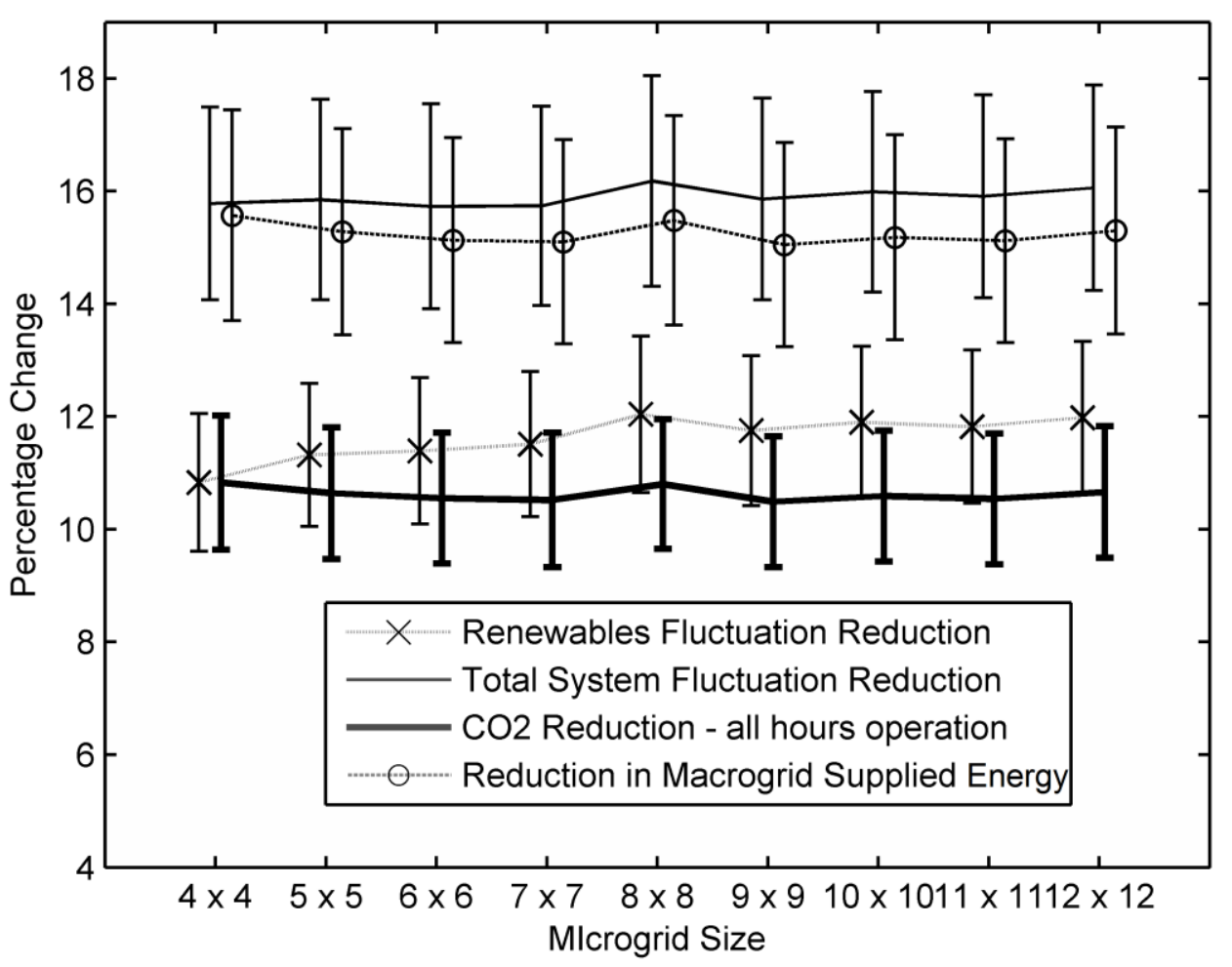

Figure 9: Change in fluctuation reduction and system output with increased microgrid size.

\subsubsection{Optimisation criteria}

Figure 10 compares outputs for two optimisations: peak hour optimisation, where the optimisation algorithm considers only peak hours of demand, which is arguably the most important challenge to achieve, and all-hour optimisation. The choice makes a big difference to the optimal system, which comprises $(82.8 \pm 9.9) \% \mathrm{PV}$ panels for peak hour optimisation and $(33.6 \pm 4.0) \%$ for all-hour optimisation. This is a result of PV panels regularly peaking in generation around midday, correlating to periods of peak demand. Wind is more intermittent and results in the turbines generating energy at times when demand may be below average. When the optimisation considers all hours of the day, this intermittency becomes less important. As fluctuations are defined as those above the average, or peak hours, the increase in fluctuation reduction during peak demand hour optimisation (Figure 10) is expected. However the reduction in energy supplied from the macrogrid is $(15.3 \pm 1.9) \%$ during peak hour optimisation, compared with $(11.9 \pm 1.4) \%$ for all hour optimisation, and $\mathrm{CO}_{2}$ emissions for all hours of operation fall from $(10.7 \pm 1.2) \%$ to $(8.0 \pm 0.9) \%$. These results indicate there is an advantage to optimising for peak hour demand times, however it should be noted that $\mathrm{CO}_{2}$ emission mitigation for renewable hours of operation remains constant, as expected. Increased $\mathrm{CO}_{2}$ mitigation benefits due to the optimised dispatch of storage linked renewables is reflected in the work by Sanseverino et al. [53]. 


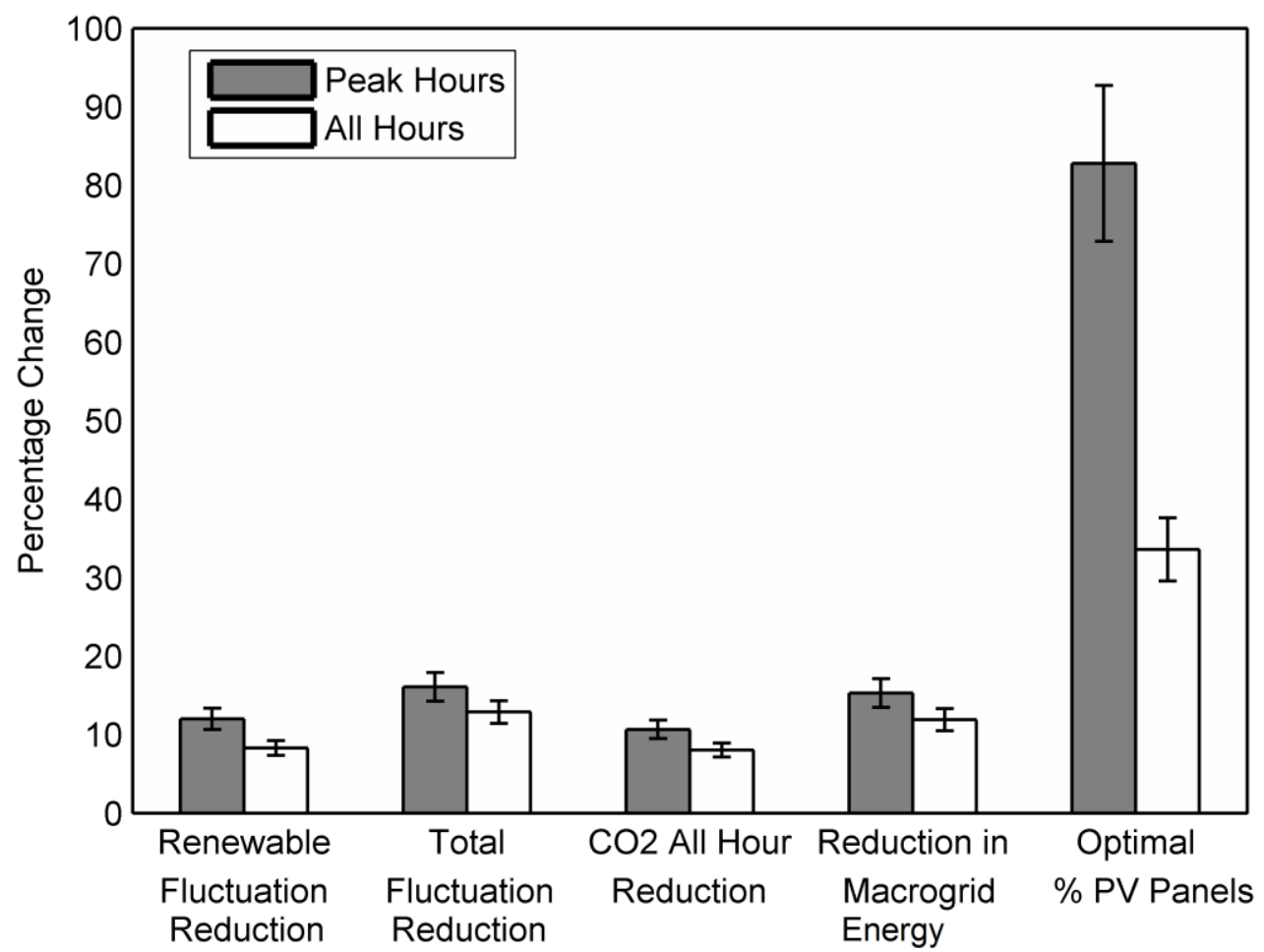

Figure 10: Comparison of system with optimisation for all hours or peak hours of operation.

In an optimised $12 \times 12$ microgrid, with one renewables unit per household, peak demand hour fluctuations reduce by $(19.3 \pm 2.2) \%$. The optimal renewables mix in the system, consisting of (82.8 \pm 9.9)\% PV panels and (17.2 \pm 2.1$) \%$ wind turbines, would reduce demand fluctuations by $(12.3 \pm 1.4) \%$, batteries by $(4.6 \pm 0.5) \%$ and DR by $(3.5 \pm 0.4) \%$. Renewable generation units have the greatest influence on demand fluctuation reduction.

\subsection{Insights from the model}

Figure 11 compares the output of the diverse microgrid (83\% PV and 17\% wind turbine, as described above) with a PV-only and turbine-only microgrid, for a peak demand hours optimisation. Diverse and PV-only systems yield similar reductions in demand fluctuation, system energy demand and emissions, with the PV-only system obtaining $\sim 97 \%$ of the reductions obtained by the diverse microgrid. In contrast, the wind turbine-only system generally returns about half the reductions, both total system energy and total demand fluctuation reduction are $53 \%$ less than the diverse microgrid, because of the significantly more intermittent output of wind turbines than PV panels. The net benefit of $\sim 3-4 \%$ reductions obtained by the diverse microgrid over the PV-only system is small, but this analysis shows that these benefits arise from the allocation of the renewable units themselves, rather than demand response or storage, with implications for microgrid design. These results are consistent with those of Naraharisetti et al. [54] who show that as diversity of generators increases demand profiles become easier to balance. 


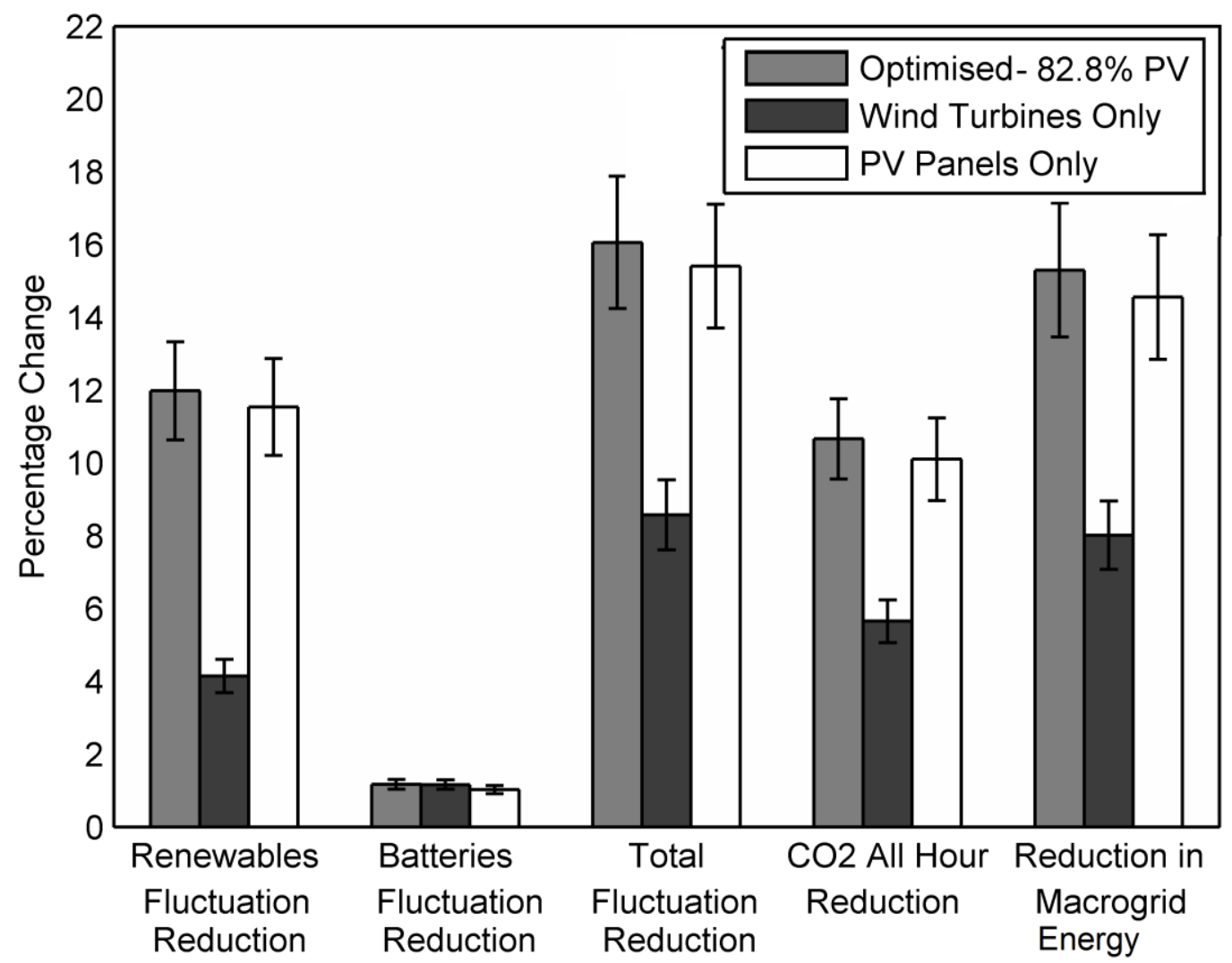

Figure 11: Comparison of system diversity to PV and turbine-only systems.

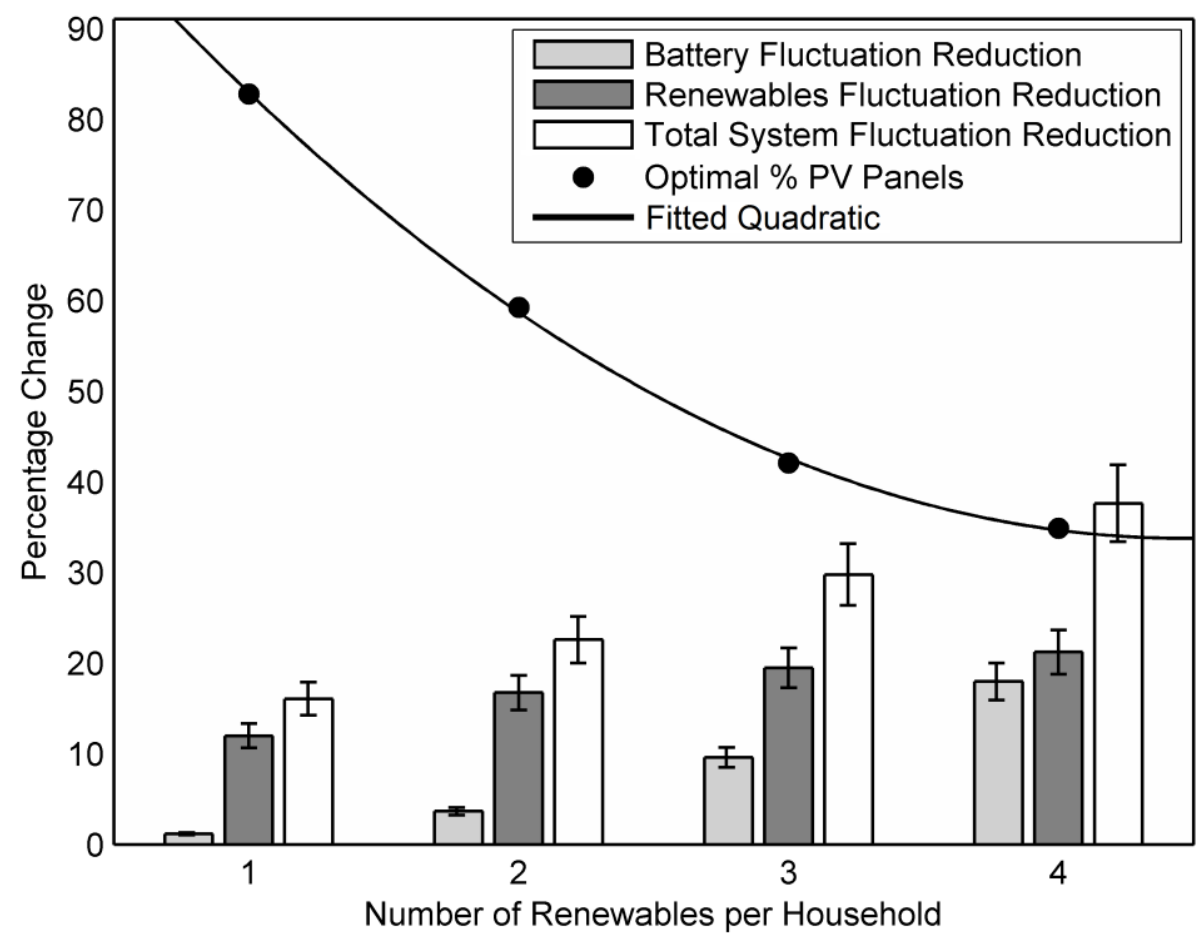

Figure 12: Change in fluctuation reduction and system configuration with increased renewable units per household. 
Figure 12 demonstrates the impact of increasing the number of renewable units per home. Increasing the number of renewable units more than doubles the total demand fluctuation reduction, from $(16.1 \pm 1.8) \%$ for one renewable unit per household to $(37.6 \pm 4.3) \%$ for four. The associated increase in energy generation raises the microgrid's reliance on storage batteries, which rises from $7 \%$ of the total fluctuation reduction for one renewable unit per household, to $48 \%$ for the system with four renewable units per household. Interestingly the ratio of PV panels to wind turbines selected by the optimization algorithm changes from $(82.8 \pm 10.0) \% \mathrm{PV},(17.2 \pm 2.1) \%$ turbines to $(34.9 \pm 4.2) \% \mathrm{PV}$ and $(62.1 \pm 7.4)$ turbines from the one to four renewable units per household scenarios. The reduction in $\mathrm{CO}_{2}$ emissions (all hours of operation) and the energy supplied by the microgrid both double with the 4-fold increase in renewable units, however the cost to benefit ratio is poor and there would be an issue of space in operationalising this system as roof space is limited.

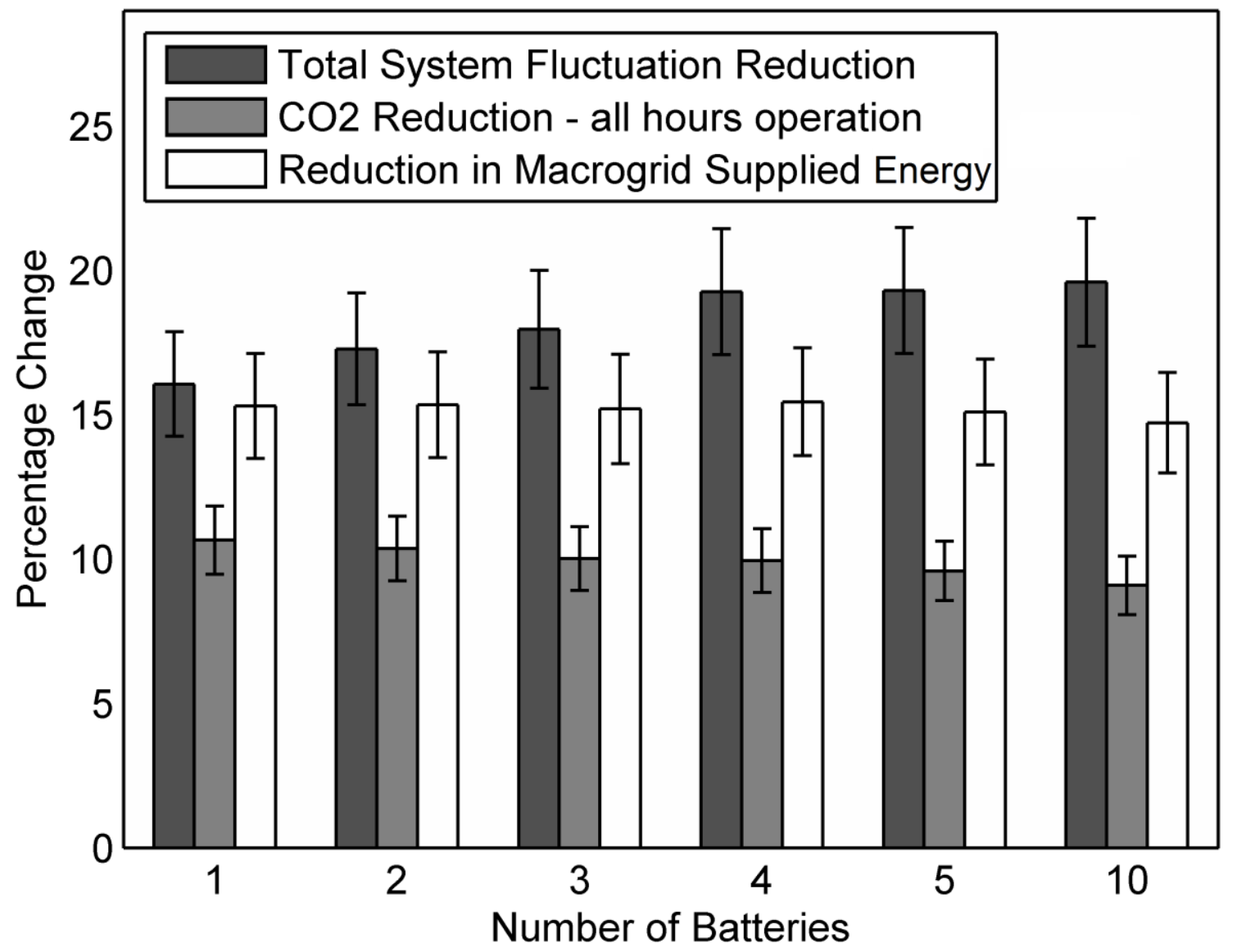

Figure 13: Change in fluctuation reduction and system output with increased number of batteries per household.

Given the growing reliance on batteries as renewables increase in the microgrid, the effects of increasing the number of batteries per household were also investigated. The model was run for systems containing up to 10 batteries and one renewable per household. As Figure 13 shows, the reduction in demand fluctuation attributable to batteries rises from $(1.2 \pm 0.1) \%$ for one battery to $(4.6 \pm 0.5) \%$ for four. Beyond this number, there was only a small change in fluctuation attributable to batteries $(5.3 \% \pm 0.6)$, and no significant change in total fluctuation reduction: $(16 \pm 2) \%$ for one battery in the system; $(19 \pm$ 
2) $\%$ for four, and (20 \pm 2$) \%$ for 10 . There was also no significant reduction in macrogrid-supplied energy. There are few instances when renewable generated energy exceeds demand, even with multiple units per household. If there were more of these instances then batteries could potentially capture otherwise wasted energy; as it is, they are used to shift energy temporally to reduce fluctuations.

\section{Conclusions}

This microgrid simulation model using real-world observational data on weather (solar irradiation, wind) and energy consumption demonstrates that including a diversity of renewable energy generation technologies and optimising the mix of renewable units substantially reduces energy balance fluctuations in a small-scale microgrid. In combination with demand reduction and storage, the fluctuations are further reduced, but the renewable units have the greatest influence on the fluctuation reduction. For the optimal 12 x 12 system with one renewable unit per household (83\% PV, 17\% wind turbines), a $12 \%$ reduction in fluctuation was achieved by optimising the renewables mix, with a further $4.6 \%$ reduction achieved by including storage batteries in the system, and 3.5\% through demand reduction. The simulated demand on macrogrid energy supply was reduced by $16 \% . \mathrm{CO}_{2}$ emissions were assessed on a life-cycle basis using previous studies LCA carbon equivalent emissions per $\mathrm{kWh}$ for each new technology relative to the current electricity baseline emissions. $\mathrm{CO}_{2}$ emissions were reduced by $(10 \pm 1) \%$ for all hours of operation, and $(74 \pm 1) \%$ during the hours of renewable supply.

Changing the size of the simulated microgrid had a surprisingly small effect on demand fluctuations and on the simplified renewables mix investigated in this study. More significant changes were seen in the optimal configuration of the microgrid when the number of renewable units per household was increased, with correspondingly more wind turbines being selected by the optimisation algorithm. While adding batteries for storage of energy does reduce fluctuations, this analysis indicated that four batteries per household with one renewable technology was optimal, after which the high embedded carbon cost of batteries, determined on a life-cycle analysis basis, reduces $\mathrm{CO}_{2}$ mitigation potential. If diversity of generation is increased, the gains in terms both of energy system stability and climate mitigation will likely become more pronounced. Other currently available elements that could be deployed in this system include combined heat and power, hydro turbine units, and community-scale technologies such as biomass burners.

The simulation model can in principle be expanded and improved by incorporating energy demand and climatic data from more varied communities than the UK urban locations investigated here. Using multiobjective linear programming techniques would allow the $\mathrm{CO}_{2}$ mitigation potential of the system to be investigated explicitly, rather than indirectly after calculating fluctuation reduction, although this is constrained by the paucity of life cycle and emissions data on renewable units. This simulation does not constitute a complete energy system; however, optimising reductions in demand fluctuations and demonstrating the mitigation potential of a diverse renewables system can help bring the independent microgrid concept a step closer.

\section{References}

[1] Sims REH, Schock RN, Adegbululgbe A, Fenhann J, Konstantinaviciute I, Moomaw W, Nimir HB, Schlamadinger B. Energy supply. In: Metz B, Davidson OR, Bosch PR, Dave R, Meyer LA, editors. Climate change 2007: Mitigation. Contribution of Working Group III to the Fourth Assessment Report of the Intergovernmental Panel on Climate Change. Cambridge University Press, 2007. 
[2] Levine M, Ürge-Vorsatz D, Blok K, Geng L, Harvey D, Lang S, Levermore G, Mehlwana AM, Mirasgedis S, Novikova A, Rilling J, Yoshino H. Residential and commercial buildings. In: Metz B, Davidson OR, Bosch PR, Dave R, Meyer LA, editors. Climate change 2007: Mitigation. Contribution of Working Group III to the Fourth Assessment Report of the Intergovernmental Panel on Climate Change. Cambridge University Press, 2007.

[3] Grubb M, Haites E, Omassoli S, Bremner C, Vincent D, Purvis N, Muller B, Butler N, Kameyama Y, Sato M, Safonov Y. Energy and climate : opportunities for the G8. Cambridge Centre for Energy Studies 2008.

[4] Stern N. Stern review: the economics of climate change. HM Treasury, London 2006.

[5] MacLeay I, Harris K, Michaels C. Digest of United Kingdom energy statistics. 2007.

[6] Ledesma P. Transient stability of a fixed speed wind farm. Renewable Energy 2003;28(9):13411355.

[7] Barton JP, Infield DG. Energy storage and its use with intermittent renewable energy. Energy 2004;19(2):441-448.

[8] Zhang W, Feliachi A. Residential load control through real-time pricing signals. Proceedings of the 35th Southeastern Symposium on System Theory, 2003. 2003:269-272.

[9] Patterson W. Full circle. Cogeneration and On-Site Power Production, London: Royal Institute of International Affairs 2000.

[10] Hawkes AD, Leach MA. Modelling high level system design and unit commitment for a microgrid. Applied Energy 2009;86:1253-1265.

[11] Wolfe P. The implications of an increasingly decentralised energy system. Energy Policy 2008;36:4509-4513.

[12] Piagi P, Lasseter RH. Autonomous control of microgrids. IEEE Power Engineering Society General Meeting 2006.

[13] Bayod-Rújula A. Future development of the electricity systems with distributed generation. Energy 2009;34(3):377-383.

[14] Jenkins N, Allan R, Crossley P, Kirschen D, Strbac G. Embedded generation. Cambridge University Press, 2000.

[15] Abusharkh S, Arnold R, Kohler J, Li R, Markvart T, Ross J, Steemers K, Wilson P, Yao R. Can microgrids make a major contribution to UK energy supply? Renewable and Sustainable Energy Reviews 2006;10(2):78-127.

[16] Lasseter RH, Paigi P. Microgrid: a conceptual solution. IEEE Annual Power Electronics Specialists Conference 2004;35:4285-4290.

[17] Lasseter RH. Microgrids and distributed generation. Journal of Energy Engineering 2007;133(3):144. 
[18] Pudjianto D, Ramsay C, Strbac G. Virtual power plant and system integration of distributed energy resources. Power 2007;1(1):10-16.

[19] Jiayi H, Chuanwen J, Rong X. A review on distributed energy resources and microgrid. Renewable and Sustainable Energy Reviews 2008;12(9):2472-2483.

[20] Lopes J, Hatziargyriou N, Mutale J, Djapic P, Jenkins N. Integrating distributed generation into electric power systems: a review of drivers, challenges and opportunities. Electric Power Systems Research 2007;77(9):1189-1203.

[21] Pepermans G, Driesen J, Haeseldonckx D, Belmans R, Dahaeseleer W. Distributed generation: definition, benefits and issues. Energy Policy 2005;33(6):787-798.

[22] Ibrahim H, Ilinca A, Perron J. Energy storage systems: characteristics and comparisons. Renewable and Sustainable Energy Reviews 2008;12(5):1221-1250.

[23] Baba J, Suzuki S, Numata S, Yonezu T, Kusagawa S, Denda A, Nitta T, Masada E. Combined power supply method for micro grid by use of several types of distributed power generation systems. European Conference on Power Electronics and Applications 2005.

[24] Sovacool BK, Watts C. Going completely renewable: is it possible (let alone desirable)? The Electricity Journal 2009;22(4):95-111.

[25] Venkataramanan G, Illindala M. Microgrids and sensitive loads. IEEE Power Engineering Society Winter Meeting. Conference Proceedings 2002:315-322.

[26] Moura PS, de Almeida AT. Multi-objective optimization of a mixed renewable system with demand-side management. Renewable and Sustainable Energy Reviews 2010;14(5):1461-1468.

[27] Ren H, Gao W. A MILP model for integrated plan and evaluation of distributed energy systems. Applied Energy 2010;87(3):1001-1014.

[28] Pecas Lopes JA, Moreira CL, Madureira AG. Defining control strategies for microgrids islanded operation. Power Systems, IEEE Transactions 2006;21(2):916-924.

[29] Chen H, Ooka R, Iwamura K, Huang H, Yoshizawa N, Miisho K, Yoshida S, Namatame S, Sakakura a, Tanaka S. Study on sustainable redevelopment of a densely built-up area in tokyo by introducing a distributed local energy supply system. Energy and Buildings 2008;40(5):782792.

[30] Kenny R, Law C, Pearce JM. Towards real energy economics: energy policy driven by lifecycle carbon emission. Energy Policy 2010;38(4):1969-1978.

[31] DEFRA. Act on $\mathrm{CO}_{2}$ calculator : public trial version. Data, Methodology and Assumptions Paper 2007.

[32] Phillips R, Blackmore P, Anderson J, Clift M, Aguilo-Rullan. A, Pester S. Micro-wind turbines in urban environments: an assessment. Building Research Establishment, Watford, UK 2007. 
[33] Renewable Devices Swift Turbines Ltd. Swift roof top wind energy system product specifications. Available at: Www.renewabledevises.com/swift/specification.htm 2005.

[34] BP Solar Ltd. Sx 3200b 200 watt photovoltaic module product specifications. Available at: Www.beyondoilsolar.com/bp3200_data_sheet.pdf 2009.

[35] Mackay JC. Sustainable energy — without the hot air. UIT Cambridge Ltd, 2009.

[36] Knight I, Kreutzer N. Three European domestic electrical consumption profiles. In: Welsh school of architecture, cardiff. IEA / ECBCS annex 42, fc+cogen-sim. 2006.

[37] Walker G. Evaluating MPPT convertor topologies using a matlab PV model. Journal of Electrical \& Electronics Engineering 2001.

[38] UK Meteorological office. MIDAS land surface stations data (1853-current), [internet].NCAS British Atmospheric Data Centre, 2006. Available from http://badc.nerc.ac.uk/view/badc.nerc.ac.uk_atom_dataent_ukmo-midas

[39] Mithraratne N. Roof-top wind turbines for microgeneration in urban houses in new zealand. Energy and Buildings 2009;41(10):1013-1018.

[40] Tahbaz M. The estimation of the wind speed in urban areas. International Journal of Ventilation 2009;8(1):75-84.

[41] Davenport A. Rational for determining design wind velocities. ASCE Journal of the Structural Division 1960;86(5):39-68.

[42] Peacock A, Jenkins D, Ahadzi M, Berry A, Turan S. Micro wind turbines in the UK domestic sector. Energy and Buildings 2008;40(7):1324-1333.

[43] Jenkins D, Fletcher J, Kane D. Model for evaluating impact of battery storage on microgeneration systems in dwellings. Energy Conversion and Management 2008;49(8):24132424.

[44] Zoka Y, Sugimoto A, Yorino N, Kawahara K, Kubokawa J. An economic evaluation for an autonomous independent network of distributed energy resources. Electric Power Systems Research 2007;77(7):831-838.

[45] Dantzig, GB, Orden A, Wolfe P. Generalized simplex method for minimizing a linear form under linear inequality constraints. Pacific J. Math 1955;5:183-195.

[46] Mehrotra S. On the implementation of a primal-dual interior point method. SIAM Journal on Optimization 1992;2:575-601.

[47] Kirby B, Hirst E. Customer specific metrics for the regulation and load-following ancillary services. Regulation 2000.

[48] Rankine RK, Chick JP, Harrison GP. Energy and carbon audit of a rooftop wind turbine. Power and Energy 2006;220:643-654. 
[49] Pacca S, Sivaraman D, Keoleian GA. Parameters affecting the life cycle performance of PV technologies and systems. Energy Policy 2007;35:3316-3326.

[50] Kemmoku Y, Ishikawa K, Nakagawa S, Kawamoto T, Sakakibara T. Life cycle $\mathrm{CO}_{2}$ emissions of a photovoltaic / wind / diesel generating system. Electrical Engineering in Japan 2002;138(2):14-23.

[51] Weisser D. A guide to life-cycle greenhouse gas emissions from electric supply technologies. Energy 2007;32:1543-1559.

[52] Rydh CJ. Environmental assessment of vanadium redox and lead-acid batteries for stationary energy storage. Journal of Power Sources 1999;80:21-29.

[53] Sanseverino ER, Silvestre MLD, Ippolito MG, Paola AD, Re GL. An execution, monitoring and replanning approach for optimal energy management in microgrids. Energy 2011;36(5):34293436.

[54] Naraharisetti PK, Karimi IA, Anand A, Lee D-Y. A linear diversity constraint - application to scheduling in microgrids. Energy 2011;36(7):4235-4243. 